\title{
Single-impulse magnetic focusing of launched cold atoms
}

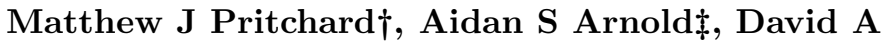 \\ Smith $\dagger$ and Ifan G Hughes $\dagger$ \\ $\dagger$ Department of Physics, Rochester Building, University of Durham, South \\ Road, Durham, DH1 3LE, UK \\ $\ddagger$ Department of Physics, University of Strathclyde, Glasgow, G4 0NG, UK \\ E-mail: i.g.hughes@durham.ac.uk
}

\begin{abstract}
We have theoretically investigated the focusing of a launched cloud of cold atoms. Time-dependent spatially-varying magnetic fields are used to impart impulses leading to a three-dimensional focus of the launched cloud. We discuss possible coil arrangements for a new focusing regime: isotropic 3D focusing of atoms with a single magnetic lens. We investigate focusing aberrations and find that, for typical experimental parameters, the widely used assumption of a purely harmonic lens is often inaccurate. The baseball lens offers the best possibility for isotropically focusing a cloud of weak-field-seeking atoms in 3D.
\end{abstract}

\section{Introduction}

The development of laser-cooling techniques [1] has facilitated the preparation of samples of atoms at microKelvin temperatures [2. It is therefore now possible to modify drastically the centre-of-mass motion of atoms, in direct contrast with the small angular deflection of fast beams studied prior to the development of laser cooling [3]. The (very strong) optical scattering force utilised to cool atoms can also be used to manipulate their position, as can the (relatively weak) conservative optical dipole force. Since the dipole force is coherent it represents a better way of manipulating cooled atoms than the scattering force (for which the random nature of absorption and spontaneous emission leads to Brownian heating). An alternative method of manipulating paramagnetic cold atoms is to use the Stern-Gerlach force 4 .

One of the goals in the field of atom optics [5] is to realise atom-optical elements that are analogues of conventional optical devices, such as mirrors, lenses and beamsplitters. An atom mirror reverses the component of velocity perpendicular to the surface and maintains the component parallel to the surface. An atom lens can modify both the transverse velocity component and the longitudinal component. To date, the Stern-Gerlach force has been used to realise flat atomic mirrors [6], curved atomic mirrors [7], and pulsed mirrors for both cold (thermal) [8] and Bose condensed atoms [9]. It has also been demonstrated that the surface of a magnetic mirror can be adapted in real time with corrugations that can be manipulated in times shorter than the atom-mirror interaction time [10].

There are many reasons for studying focusing of launched cold atoms: atom lithography [11]; transferring cold atoms from a MOT to a remote vacuum chamber of 
lower background pressure 12]; loading miniature magnetic guides [13, atom chips [14] and storage rings [15]. In comparison to an unfocused cloud, the density of the cloud can be increased by many orders of magnitude after magnetic focusing.

The first demonstration of 3D focusing using pulsed magnetic lenses was conducted by Cornell et al. [16. However, their work did not address the optimum strategy for achieving a compact focused cloud, nor did they discuss the limiting features for the quality of their atom-optical elements. The group of Gorceix has performed experiments demonstrating the longitudinal Stern-Gerlach effect with an atomic cloud using pulsed magnetic forces [17, and an experimental and theoretical study of cold atom imaging by means of magnetic forces [18. To date, the experimental and theoretical studies of pulsed magnetic focusing have been analysed under the assumption that the magnetic lens potential is harmonic - this work addresses the validity of this approximation, and the effects of aberrations. We note also that there has been no theoretical or experimental work on full 3D focusing of cold atoms using a single magnetic pulse, although this is a very useful focusing strategy.

The scope of this paper is to investigate theoretically and numerically the limiting factors to the quality and size of the final image obtained in pulsed magnetic focusing experiments; to identify the sources of aberration; and to discuss schemes for minimising their deleterious effect. In this paper we restrict our attention to focusing strategies using a single magnetic impulse. A second paper describing more than one impulse (alternate gradient focusing) [19] is in preparation. Whilst this work shall concentrate on the analysis for achieving a compact cloud in space, it is also possible to use pulsed magnetic fields to reduce the momentum spread of an expanding cloud with appropriate magnetic impulses. This can be viewed as an implementation of $\delta$ kick cooling, which has been demonstrated with atoms [20, ions [21] and Bose Einstein condensates (BEC) 9]. Some of the techniques described here are also successfully used for the deceleration of polar molecules using time-varying electric fields [22]. Atom-optical elements realised with light forces [1, 23] are beyond the scope of this paper.

The remainder of the paper is organised as follows: Section 2 outlines the theory of how to achieve the desired magnetic fields; Section 3 contains an analysis of magnetic imaging and minimising the final cloud size; Section 4 describes and contrasts the performance of different magnetic lenses; Section 5 contains a discussion and concluding remarks.

\section{Magnetic lens theory}

\subsection{The Stern-Gerlach force}

An atom's magnetic dipole interaction energy is $U=m_{F} g_{F} \mu_{\mathrm{B}} B$ for a field of magnitude $B$, where $m_{F}$ is the magnetic quantum number, $g_{F}$ is the Landé g-factor and $\mu_{\mathrm{B}}$ the Bohr magneton. We assume that field zeros are avoided and that the magnetic moment adiabatically follows the field. Depending on an atom's hyperfine quantum state, the Stern-Gerlach force $F=-\nabla U \propto \nabla B$ can be used to attract it towards weak (if $m_{F} g_{F}>0$ ) or strong (if $m_{F} g_{F}<0$ ) magnetic fields. The choice

of whether atoms in weak or strong-field seeking states are launched depends on the particular application. For some applications, e.g. loading a remote dipole trap, or a secondary magneto-optical trap, it does not matter which atomic state is used. In this work we assume that the ensemble (of alkali metal atoms) has been optically 
pumped into the stretched state $\left|F=I+1 / 2, m_{F}=F\right\rangle$ (a weak-field seeking state with $\left.m_{F} g_{F}=1\right)$.

A purely harmonic magnetic field magnitude will result in an aberration-free lens. In this paper we will consider current-carrying wires assembled to give two different kinds of second order magnetic field magnitude:

$$
\begin{aligned}
& B_{1 \mathrm{D}}(x, y, z)=B_{0}+\frac{B_{2}}{2}\left(-x^{2} / 2-y^{2} / 2+\left(z-z_{\mathrm{c}}\right)^{2}\right), \\
& B_{3 \mathrm{D}}(x, y, z)=B_{0}+B_{1}\left(z-z_{\mathrm{c}}\right)+\frac{B_{2}}{2}\left(x^{2}+y^{2}+\left(z-z_{\mathrm{c}}\right)^{2}\right) .
\end{aligned}
$$

$B_{0}, B_{1}$ and $B_{2}$ are the bias field, the axial gradient and the field curvature, respectively. In both cases these parameters are chosen to prevent the field magnitude exhibiting zeros in the region of interest, thus avoiding Majorana spin-flip transitions [24. The point $\left\{0,0, z_{c}\right\}$ defines the centre of the lens. A lens of the form of $B_{1 \mathrm{D}}$ can be used either to focus axially or radially; a lens of the form of $B_{3 \mathrm{D}}$ is used to focus isotropically in $3 \mathrm{D}$. The accelerations associated with these fields are harmonic about $\left\{0,0, z_{\mathrm{c}}\right\}$ :

$$
\begin{aligned}
& \mathbf{a}_{1 \mathrm{D}}=-\omega^{2}\left\{-x / 2,-y / 2,\left(z-z_{c}\right)\right\}, \\
& \mathbf{a}_{3 \mathrm{D}}=-\omega^{2}\left\{x, y,\left(z-z_{c}\right)\right\}+\mathbf{a}_{0},
\end{aligned}
$$

where $\omega^{2}=\mu_{\mathrm{B}} B_{2} / m$ is a useful measure of the power of the lens, $\mathbf{a}_{0}=\left\{0,0, \mu_{\mathrm{B}} B_{1} / m\right\}$ is a constant acceleration arising from axial magnetic gradients, and $m$ is the atomic mass. Note that for the $1 \mathrm{D}$ case (equation (3)) the axial curvature is twice the magnitude of and opposite in sign to the radial curvature, $\omega_{\mathrm{r}}^{2}=-2 \omega_{\mathrm{z}}^{2}$.

The acceleration of equation (3) results in a lens which is axially converging (diverging) and radially diverging (converging) if $B_{2}$ is positive (negative). In order to achieve a $3 \mathrm{D}$ focus with such lenses, an axially converging lens pulse must be followed by an appropriately timed axially diverging lens (or vice versa). This alternategradient focusing strategy is the subject of another paper [19]. In contrast, this paper details novel lenses which yield accelerations of the form in equation (44). Isotropic 3D focusing can be achieved with a single lens pulse for atoms in weak-field-seeking states, as Earnshaw's theorem (which states that a maximum of magnetic field magnitude in free space is not allowed [25]) ensures $B_{2} \geq 0$ in this case. Note that the harmonic accelerations in both equation (3) and (44) lead to three separable one-dimensional simple harmonic oscillator equations for the atomic motion.

\subsection{Magnetic fields from current bars and circular coils}

This work considers straight current bars and circular coils for the formation of lenses. The Biot-Savart law yields magnetic fields that are analytic for both finiteand infinite-length current bars. For circular coils the field can be expressed in terms of elliptic integrals [26]. A discussion of the form of the contours of magnetic field magnitude for various magnetic trapping configurations has been provided by Bergeman et al. 27. The fields are constrained by Maxwell's equations, which, in conjunction with symmetry arguments, allow the spatial dependence of the fields to be parameterised with a small number of terms. In particular, for a cylindrically symmetric magnetic coil configuration, the fourth-order on-axis 1D magnetic field, $B_{z}(r=0, z)=\sum_{i=0}^{4} B_{i} z^{i} / i$ !, gives the complete fourth-order 3D magnetic field:

$$
\mathbf{B}(r, z)=\left\{B_{r}, B_{z}\right\}
$$




$$
\begin{aligned}
& =\left\{-\frac{B_{1}}{2} r-\frac{B_{2}}{2} r z, B_{0}+B_{1} z+\frac{B_{2}}{2}\left(z^{2}-\frac{r^{2}}{2}\right)\right\}+ \\
& B_{3}\left\{\frac{r^{3}}{16}-\frac{r z^{2}}{4}, \frac{z^{3}}{6}-\frac{r^{2} z}{4}\right\}+B_{4}\left\{\frac{r^{3} z}{16}-\frac{r z^{3}}{12}, \frac{z^{4}}{24}-\frac{r^{2} z^{2}}{8}+\frac{r^{4}}{64}\right\},
\end{aligned}
$$

with third-order magnitude:

$$
\begin{aligned}
B(r, z)= & B_{0}+B_{1} z+\frac{1}{2} B_{2} z^{2}+\frac{1}{2}\left(\frac{B_{1}{ }^{2}}{4 B_{0}}-\frac{B_{2}}{2}\right) r^{2} \\
& +B_{3} \frac{z^{3}}{6}+r^{2} z\left(-\frac{B_{3}}{4}+\frac{B_{1} B_{2}}{4 B_{0}}-\frac{B_{1}{ }^{3}}{8 B_{0}{ }^{2}}\right) .
\end{aligned}
$$

If, in addition, the coil system is axially-symmetric the magnitude to fourth order is:

$$
B(r, z)=B_{0}+\frac{1}{2} B_{2}\left(z^{2}-r^{2} / 2\right)+B_{4}\left(\frac{z^{4}}{24}+\frac{r^{4}}{64}\right)+\frac{r^{2} z^{2}}{8}\left(-B_{4}+\frac{B_{2}^{2}}{B_{0}}\right) .
$$

Let us consider two coils of $N$ turns with radius $a$, separation $s$, carrying currents $I_{1}$ and $I_{2}$. It is convenient to partition the currents in each coil as a current $I_{\mathrm{H}}$ with the same sense and a current $I_{\mathrm{AH}}$ in opposite senses, i.e. $2 I_{\mathrm{H}}=I_{1}+I_{2}, 2 I_{\mathrm{AH}}=I_{1}-I_{2}$. If one defines $\eta=\mu_{0} N I / 2$, and uses the scaled separation $S=s / a$, the axial magnetic field is thus:

$$
B(0, z)=\left(\frac{\left(\eta_{\mathrm{H}}+\eta_{\mathrm{AH}}\right)}{a\left(1+(z / a-S / 2)^{2}\right)^{3 / 2}}+\frac{\left(\eta_{\mathrm{H}}-\eta_{\mathrm{AH}}\right)}{a\left(1+(z / a+S / 2)^{2}\right)^{3 / 2}}\right),
$$

yielding the axial Taylor expansion terms:

$$
\begin{aligned}
& B_{0}=\frac{2 \eta_{\mathrm{H}}}{a\left(1+S^{2} / 4\right)^{\frac{3}{2}}}, \quad B_{1}=\frac{3 \eta_{\mathrm{AH}} S}{a^{2}\left(1+S^{2} / 4\right)^{\frac{5}{2}}}, \quad B_{2}=\frac{6 \eta_{\mathrm{H}}\left(S^{2}-1\right)}{a^{3}\left(1+S^{2} / 4\right)^{\frac{7}{2}}} \\
& B_{3}=\frac{15 \eta_{\mathrm{AH}} S\left(S^{2}-3\right)}{a^{4}\left(1+S^{2} / 4\right)^{\frac{9}{2}}}, \quad B_{4}=\frac{45 \eta_{\mathrm{H}}\left(S^{4}-6 S^{2}+2\right)}{a^{5}\left(1+S^{2} / 4\right)^{\frac{11}{2}}}
\end{aligned}
$$

Thus axially symmetric lens systems (equation (17): $\eta_{A H}=0, B_{\text {odd }}=0$ ), consisting of one or two circular coils have radial and axial strengths:

$$
\omega_{z}^{2}=-2 \omega_{r}^{2}=\frac{3 \mu_{0} \mu_{B} N I(1+\operatorname{sign}(S))\left(S^{2}-1\right)}{2 m a^{3}\left(1+S^{2} / 4\right)^{7 / 2}} .
$$

The sign function switches between a single and a double coil. The expressions of equation (9) will be utilised in the next section where various configurations of coils and bars for realising magnetic lenses are considered.

\subsection{Configurations for realising magnetic lenses}

There are six distinct coil and current bar configurations used in this paper for single-pulse focusing. Figure 1 displays these six strategies, whilst figure 2 shows the associated magnetic field information. Strategies I-III lead to the axial/radial focusing of equation (3), whereas strategies IV-VI yield isotropic 3D focusing (equation (44)). Strategies I-V deal with cylindrically symmetric coil arrangements. 


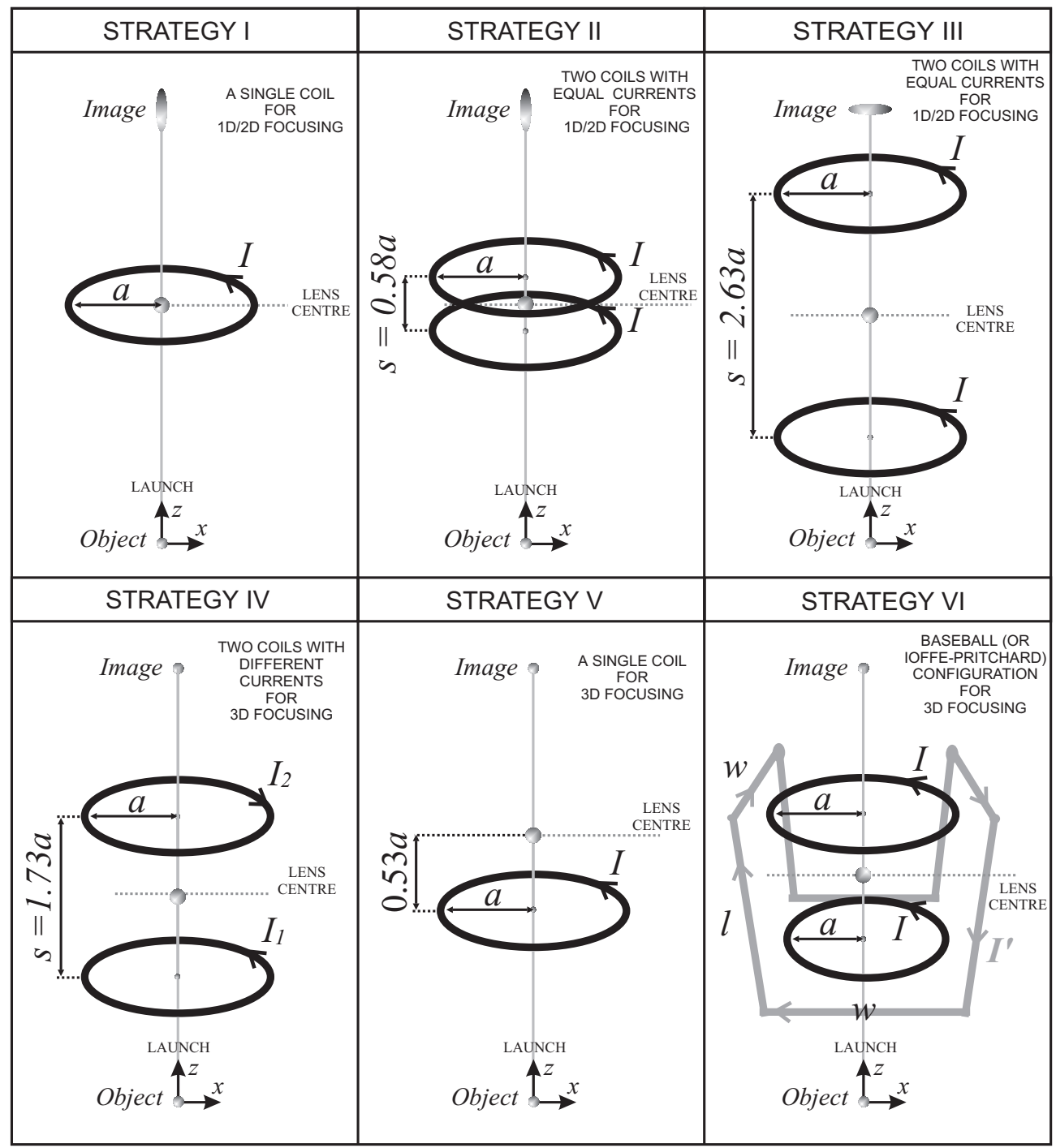

Figure 1. The six different lens strategies detailed in the text. Strategy I utilises the centre of a single coil; Strategies II and III use the geometric centre of a pair of coaxial coils (carrying equal currents in the same sense) with separations of $S=0.58$ and $S=2.63$ coil radii, respectively; Strategy IV uses the geometric centre of a pair of coaxial coils with unequal currents and a relative separation $S=\sqrt{3}$; Strategy V uses a single coil axially offset to $z / a= \pm \sqrt{2 / 7}$; Strategy VI uses the geometric centre of a Baseball coil with dimensions $w=l=2 a$ combined with a coaxial coil pair with $S=1$.

2.3.1. Strategy I: a single coil The magnetic field magnitude at the centre of a single coil of radius $a$ with $N I_{1}$ current turns is characterised by the coefficients in equation (9) with $S=0$ and using $I_{2}=0$ when evaluating $\eta_{\mathrm{H}}$. The radial curvature is positive, thus, a single coil can be used to radially focus weak-field seeking atoms. However this lens has an axial curvature that is negative and twice the magnitude. 


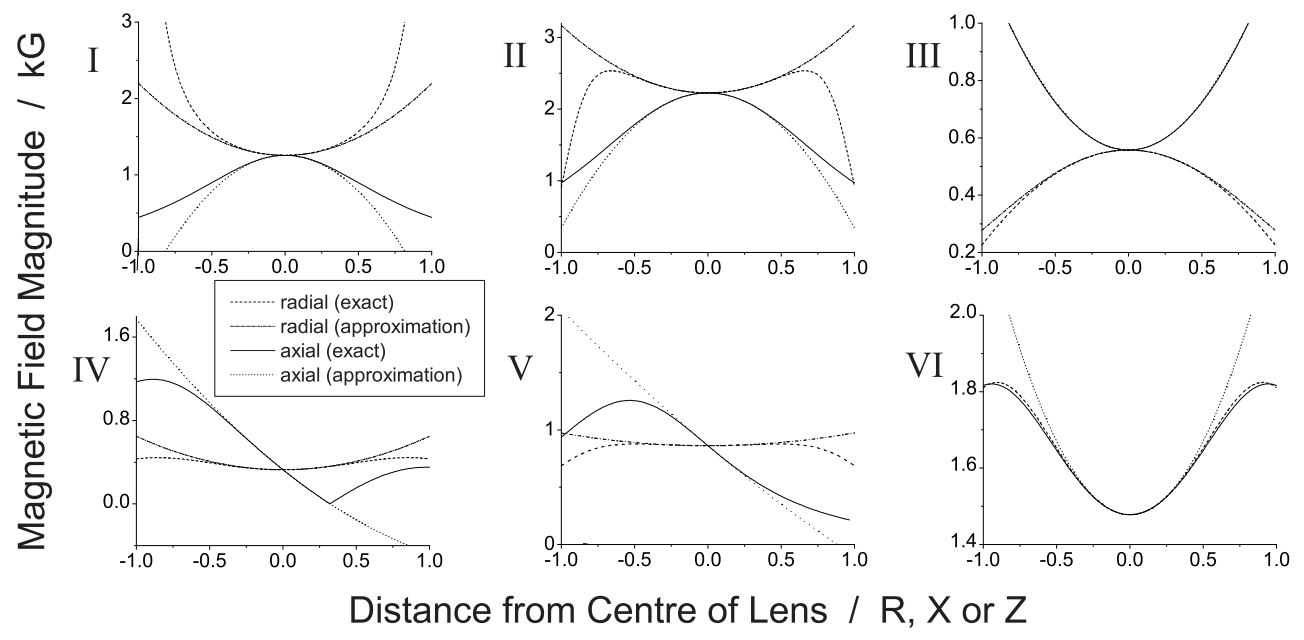

Figure 2. Here we show the respective axial and radial variation of the magnetic field magnitude associated with the six lens strategies I-VI of Figure 1$] R, X$ and $Z$ are the scaled radial, $x$ and axial displacements from the centre of the lens, respectively. In Images IV and $\mathrm{V}$ the axial gradients in the magnetic field magnitude alter the centre-of-mass velocity of an atom cloud but do not lead to lensing. Parabolic approximations to the field magnitudes are also given.

Figure 1Strategy I shows the geometry of this lens.

2.3.2. Strategies II, III and IV: a pair of axially-displaced coils For a pair of separated coaxial coils, where both coils have equal current with the same sense, an axially diverging (converging) lens is realised for separations less (greater) than the coil radius (equation (10)). The lens curvature is zero when the separation equals the coil radius (the Helmholtz condition for achieving uniform fields). The axial curvature is a factor of -2 greater than the radial curvature.

Since strategies II and III are axially-symmetric configurations there are no third order terms. The third term in equation (7) is zero when $B_{4}=0$, which occurs if $S=\sqrt{3 \pm \sqrt{7}}=0.595$ or 2.38. The fourth term in equation (7) is zero when $B_{4} B_{0}=B_{2}^{2}$, which occurs if $S=\sqrt{\frac{1}{3}(13 \pm \sqrt{145})}=0.565$ or 2.89 . The harmonicity of a radial-focusing lens ( $S<1$ Strategy II) is thus optimised if the relative coil separation is $S=0.58$, whereas the harmonicity of an axial-focusing lens $(S>1$ Strategy III) is thus optimised if the relative coil separation is $S=2.63$. Figure 1 II and III show the geometry of these optimised lenses.

Figure 1Strategy IV shows the geometry of an isotropic 3D lens formed from two coils carrying different currents. From Equation (6) we see that an isotropic 3D lens is formed when $B_{1}{ }^{2}=6 B_{2} B_{0}$, and this can be re-expressed using equation (9) as:

$$
\eta_{\mathrm{AH}}= \pm \eta_{\mathrm{H}} \sqrt{8\left(1-1 / S^{2}\right)} .
$$

From equations (69) we see that some of the third-order terms in the magnetic field magnitude are removed by setting $S=\sqrt{3}$ and thus $B_{3}=0$. This means that the coils carry currents of $I_{1}$ and $I_{2}=-0.396 I_{1}$ respectively. Note that the only way to remove all third-order terms is to have $S=\sqrt{3}$ and set $B_{1}{ }^{2}=2 B_{2} B_{0}$ (i.e. 
$\left.\eta_{\mathrm{AH}}= \pm \eta_{\mathrm{H}} \sqrt{8 / 3\left(1-1 / S^{2}\right)}\right)$ which corresponds to a purely axial lens. Purely radial lenses are achieved when $B_{2}=0$, (i.e. $S=1$ ) for non-zero $\eta_{\mathrm{AH}}$ and $\eta_{\mathrm{H}}$.

2.3.3. Strategy V: an axially offset single coil At the centre of a single coil the radial and axial curvatures have opposite signs (Strategy I), however, the radial and axial curvatures have different $z$-dependence, (Figure 1 Strategy V). At the two axial locations $z= \pm \sqrt{2 / 7}$ a the curvatures are equal in magnitude and both positive. Therefore an impulse applied to a cloud whose centre-of-mass is at either of these positions will lead to isotropic 3D focusing.

2.3.4. Strategy VI: Ioffe-Pritchard configuration Ioffe-Pritchard (IP) traps are used extensively for atom trapping and are similar to the Ioffe configuration utilised in plasma confinement [28. One of the simplest forms of this trap is with a pair of coaxial coils to generate both a bias field, $B_{0}$, and an axial curvature $B_{2}$. Four current bars run parallel to the $z$-axis, with each bar running through the corner of a square (with side length $w=W a$ ) centred on the axis. The magnitude of the current in each bar is equal, but neighbouring bars have the opposite current sense.

This configuration is not strictly radially symmetric, although it is an excellent approximation for small displacements from the axis. The four current bars give rise to a transverse magnetic field of the form $\left\{B_{x}, B_{y}\right\}=B_{1}^{\prime}\{x,-y\}$ close to the axis. Here, $B_{1}^{\prime}=4 \mu_{0} I^{\prime} /\left(\pi w^{2}\right)$, where $I^{\prime}$ is the magnitude of the current in a bar, and the prime distinguishes it from the axial gradient defined previously. The shape of the magnetic field magnitude contours depend on the geometry of the trap and the ratio of the coil current to the bar current. Such traps are extensively used in Bose-Einstein condensation experiments, where an additional circular coil pair is used primarily to lower the bias field $B_{0}$, yielding an anisotropic harmonic-oscillator potential, with tight radial confinement $B_{2 r}=B_{1}^{\prime 2} / B_{0}-B_{2} / 2$, and weaker axial confinement $B_{2 z}=B_{2}$. For the purposes of magnetic focusing an isotropic IP trap is required. This is achieved if the following relationship holds among the lens parameters:

$$
\frac{I^{\prime}}{N I}=3 \pi W^{2} \sqrt{\frac{S^{2}-1}{\left(2+S^{2} / 2\right)^{5}}}
$$

In BEC experiments variants of the IP trap described above are used, since infinitely-long current bars are not realisable. An elegant winding pattern is the baseball geometry. A baseball coil has an axial curvature in addition to a radial gradient, and these quantities can no longer be independently varied. Figure@Strategy VI shows the cuboidal baseball geometry, where the sides have lengths $w=W a$, $w=W a$ and $l=L a,\left(l\right.$ is along the $z$-axis). The bars carry a current $I^{\prime}$. It is impossible to realise a $3 \mathrm{D}$ isotropic lens solely by adjusting the aspect ratio of the coils $w / l$, and for this reason we add a coaxial pair of coils carrying equal currents in the same sense (if $\eta_{\mathrm{AH}} \neq 0$ then the equality of the $x$ and $y$ curvatures is broken). The magnetic field magnitude has a third-order Taylor expansion:

$$
B=B_{\mathrm{f} 0}+\left(\frac{B_{1}^{\prime 2}}{2 B_{\mathrm{f} 0}}-\frac{B_{\mathrm{f} 2}}{4}\right)\left(x^{2}+y^{2}\right)+\frac{B_{\mathrm{f} 2}}{2} z^{2}+\left(B_{3}^{\prime}-\frac{B_{\mathrm{f} 2} B_{1}^{\prime}}{2 B_{\mathrm{f} 0}}\right)\left(y^{2}-x^{2}\right) z,
$$

where dashed terms indicate contributions solely from the baseball coil, and the subscript $\mathrm{f}$ is used when one must add together the $B_{0}, B_{2}$ Taylor contributions from 
the circular coils (equation (9)) and $B_{0}^{\prime}, B_{2}^{\prime}$ from the following baseball coil Taylor terms:

$$
\begin{gathered}
B_{0}^{\prime}=\frac{\frac{4 \mu_{0} I^{\prime}}{\pi} W^{2}}{a\left(L^{2}+W^{2}\right) \sqrt{L^{2}+2 W^{2}}}, B_{1}^{\prime}=\frac{\frac{4 \mu_{0} I^{\prime}}{\pi}\left(L^{5}+3 L^{3} W^{2}+4 L W^{4}\right)}{a^{2} W^{2}\left(L^{2}+W^{2}\right)^{2} \sqrt{L^{2}+2 W^{2}}} \\
B_{2}^{\prime}=\frac{\frac{16 \mu_{0} I^{\prime}}{\pi}\left(6 L^{6} W^{2}+18 L^{4} W^{4}+11 L^{2} W^{6}-5 W^{8}\right)}{a^{3}\left(L^{2}+W^{2}\right)^{3}\left(L^{2}+2 W^{2}\right)^{\frac{5}{2}}}, \\
B_{3}^{\prime}=\frac{\frac{48 \mu_{0} I^{\prime}}{\pi}\left(-5 L^{7} W^{2}-10 L^{5} W^{4}+11 L^{3} W^{6}+24 L W^{8}\right)}{a^{4}\left(L^{2}+W^{2}\right)^{4}\left(L^{2}+2 W^{2}\right)^{\frac{5}{2}}} .
\end{gathered}
$$

The simplest isotropic lens to calculate is when we use circular Helmholtz coils i.e. $S=1, B_{2}=0$ and the only contribution of the circular coils in equation (13) is the axial constant field $B_{0}$, which we tune to enable lens isotropy. The finite-length current bars lead to a non-trivial relationship among the parameters for realising an isotropic lens by adjusting the circular coil bias-field and curvature. For the purpose of our calculations we use the parameters $W=W=L=2$ for a cubic baseball, with $S=1$ and a relative circular coil current of $\frac{N I}{I^{\prime}}=0.154$.

\subsection{The harmonicity of the magnetic lenses}

The previous subsection was a description of the way to achieve a radially converging lens with a single coil or a pair of coils; an axially converging lens with a pair of displaced coils; and three geometries for achieving an isotropic 3D lens. Aberrations are caused by departures of the actual potential from the ideal harmonic potential. To measure the degree of departure from harmonicity of a lens, as a function of the distance from the lens' centre, we use:

$$
\epsilon=\frac{\left|\mathbf{a}_{\mathrm{F}}-\mathbf{a}_{\mathrm{H}}\right|}{\left|\mathbf{a}_{\mathrm{H}}-\mathbf{a}_{0}\right|},
$$

where $\mathbf{a}_{\mathrm{H}}$ is the harmonic fit, (equation (3) or (44)), to the full Biot-Savart lens acceleration $\mathbf{a}_{\mathrm{F}}$. The departure from harmonicity is a function of position, and the cylindrical co-ordinates $R$ and $Z$ are used to plot $\epsilon(R, Z)$ for different coil systems. Note the scaled co-ordinates $R$ and $Z$ are normalised to measure length in units of the coil radius, $R=r / a$ and $Z=z / a$. Figure (3) shows the departure from harmonicity for the six different focusing strategies.

2.4.1. Strategies I-III: axial/radial focusing Figures 3I-III show the spatial variation of the departure from harmonicity of Strategies I-III. For Strategy I, II and III the departure from harmonicity averaged over a sphere of radius $0.25 a$ is $0.058,0.007$ and 0.009 respectively. The radially converging lens of Strategy II, and the axially converging lens of Strategy III are almost an order of magnitude more harmonic than the single coil radially converging lens of Strategy I.

2.4.2. Strategies IV-VI: isotropic 3D focusing The isotropic 3D lens of Strategy IV rapidly becomes anharmonic as one moves axially away from the lens centre because of the axial magnetic field zero illustrated in Figure 2IV. In both Strategy IV and V the lack of axial symmetry means that there are first-order terms in the field magnitude, resulting in a gradient which affects the centre-of-mass motion of the atomic cloud. 

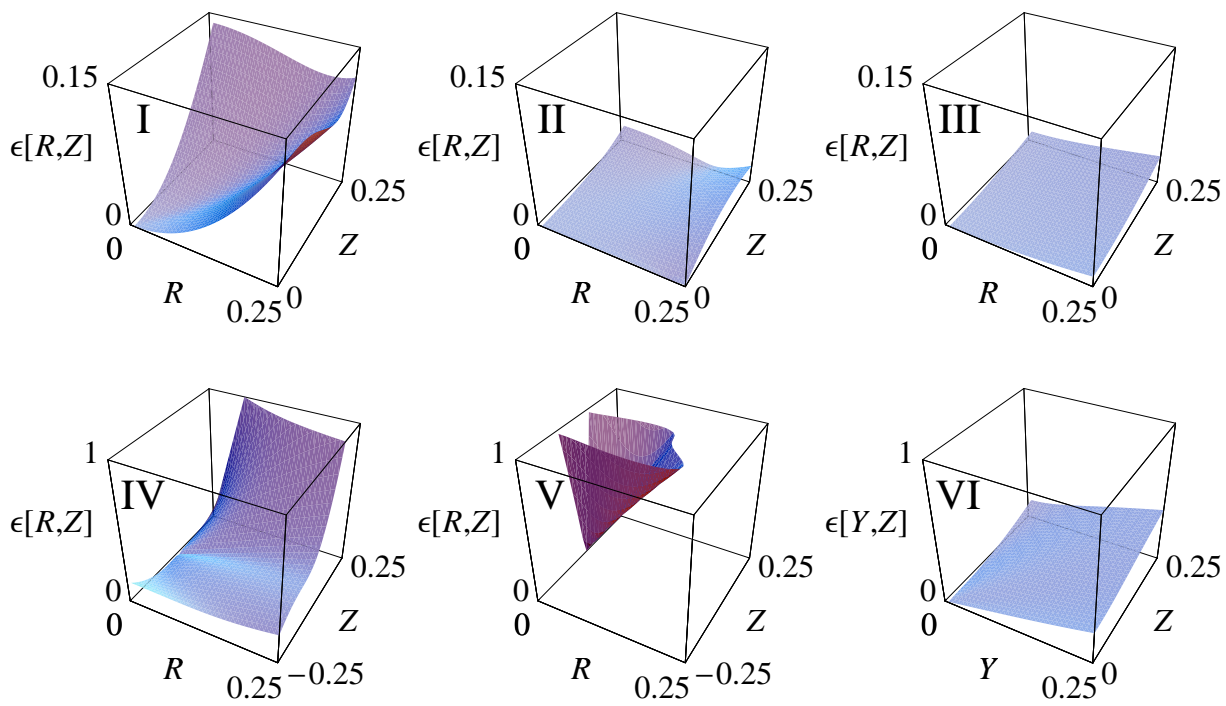

Figure 3. The departure from harmonicity $\epsilon(R, Z)$ of the six different lens strategies of Fig. 1 is considered for displacements of up to 0.25 coil radii. Averaged over a sphere of 0.25 times the coil radius, the departures from harmonicity for the six configurations are: $0.058,0.007,0.009,0.385,1.347$ and 0.148 respectively.

The main problem with Strategy V is that although both the axial and radial curvatures are equal at the lens centre, they vary rapidly with position. The lenscentre curvatures are also 8.4 times weaker than the axial curvature at the centre of a single coil, leading to longer duration magnetic lensing impulses. The cloud of atoms therefore experiences the lens' axial anharmonicities for a greater period, again compromising the quality of the focus.

Figures 3 IV-VI show the spatial dependence of the departure from harmonicity of Strategies IV-VI. For Strategy IV, V and VI the departure from harmonicity averaged over a sphere of radius $0.25 a$ is $1.347,0.385$ and 0.148 respectively. The IP lens of Strategy VI is thus significantly more harmonic than the two coil lens of Strategy IV, which is in turn considerably better than the axially offset single coil lens of Strategy V.

\section{Imaging and minimum cloud size}

The separable equations of motion for a lens that is harmonic in 3D (equations (3),(4) ) allow us to consider motion in each cartesian dimension as a separate simple harmonic equation. It is useful to employ the $\mathcal{A B C D}$-matrix formulation used widely in geometrical optics. The position and velocity of an atom along a given Cartesian axis, say $x$, is written as a 2 -component vector, and the final and initial vectors are related via the equation:

$$
\left(\begin{array}{c}
x_{\mathrm{f}} \\
v_{x_{\mathrm{f}}}
\end{array}\right)=\left(\begin{array}{cc}
\mathcal{A} & \mathcal{B} \\
\mathcal{C} & \mathcal{D}
\end{array}\right)\left(\begin{array}{c}
x_{\mathrm{i}} \\
v_{x_{\mathrm{i}}}
\end{array}\right) .
$$


To simplify the effects of gravity we perform the calculations in a free-falling frame of reference. In this frame the free evolution of the cloud is an isotropic expansion, described by an $\mathcal{A B C D}$ matrix. The influence of a converging or diverging magnetic lens can also be described by $\mathcal{A B C D}$ matrices, as outlined in [18]:

$$
M_{1}(t)=\left(\begin{array}{ll}
1 & t \\
0 & 1
\end{array}\right), M_{2}=\left(\begin{array}{cc}
\cos \omega \tau & \frac{1}{\omega} \sin \omega \tau \\
-\omega \sin \omega \tau & \cos \omega \tau
\end{array}\right), M_{3}=\left(\begin{array}{cc}
\cosh \omega \tau & \frac{1}{\omega} \sinh \omega \tau \\
\omega \sinh \omega \tau & \cosh \omega \tau
\end{array}\right) \cdot(17
$$

Matrix $M_{1}$ is the translation matrix for a duration $t ; M_{2}$ is the matrix for a converging lens of strength $\omega$ applied for a duration $\tau ; M_{3}$ is the matrix for a diverging lens of strength $\omega$ applied for a duration $\tau$. It is interesting to note that the sinusoidal (exponential) path taken by atoms inside a converging (diverging) magnetic lens is in stark contrast to the linear propagation of light rays in an optical lens.

By multiplying these matrices together, we arrive at the final $\mathcal{A B C D}$ system matrix. An image (i.e. a one-to-one map of position between the initial and final cloud) is formed if the condition $\mathcal{B}=0$ is maintained. In this case the spatial magnification $\mathcal{A}$ is the inverse of the velocity magnification $\mathcal{D}$. This spatial compression and concomitant velocity spread is a manifestation of Liouville's theorem. The theorem states that phase-space density is conserved in a Hamiltonian system. Timedependent Stern-Gerlach forces satisfy the criteria for Liouville's theorem to be valid [29]. The cloud extent along $x$ in a given plane is given by:

$$
\sigma_{x_{f}}^{2}=\left(\mathcal{A} \sigma_{x_{i}}\right)^{2}+\left(\mathcal{B} \sigma_{v_{x_{i}}}\right)^{2},
$$

where $\sigma_{x_{i}}$ is the initial position standard deviation and $\sigma_{v_{x_{i}}}$ is the initial velocity standard deviation. An image is formed for the condition $\mathcal{B}=0$, but the smallest cloud size does not necessarily occur in the same plane. For a single lens system, the minimum cloud size occurs very close to the imaging plane. For multi-lens systems, the image plane and the minimum cloud size do not necessarily correspond.

\subsection{Imaging solutions}

We note here that the 'thick' converging lens $M_{2}$ is identical to a thin lens of strength $\mathcal{C}(\omega, \tau)=-\omega \sin (\omega \tau)$ (i.e. the original $\mathcal{C}$ entry of the $M_{2} \mathcal{A B C D}$ matrix), pre- and post-multiplied by a translation matrix with duration $\tau^{\prime} / 2$ :

$$
M_{2}=\left(\begin{array}{cc}
1 & \tau^{\prime} / 2 \\
0 & 1
\end{array}\right)\left(\begin{array}{ll}
1 & 0 \\
\mathcal{C} & 1
\end{array}\right)\left(\begin{array}{cc}
1 & \tau^{\prime} / 2 \\
0 & 1
\end{array}\right) .
$$

The pulse width $\tau^{\prime}$ is defined as

$$
\tau^{\prime}(\omega, \tau)=\frac{2}{\omega} \tan \frac{\omega \tau}{2}
$$

and the notation of primes is used to denote times in the 'thin' lens representation. This means that we can use many of the simplicities of 'thin' lens optics, even if we are in fact dealing with the more accurate 'thick' lensing behaviour. The effective 'thin lens' duration of the pulse $\tau^{\prime}$ differs from the actual pulse duration $\tau$, but otherwise the treatments are identical. In the limit of a short, strong pulse $\omega \tau \rightarrow 0$, we find that $\tau^{\prime} \rightarrow \tau$. If we wish to consider the diverging lens $M_{3}$, we merely make the transformation $\omega \rightarrow i \omega$ in equation (19) - i.e. $\mathcal{C}=\omega \sinh (\omega \tau)$ and $\tau^{\prime}=\frac{2}{\omega} \tanh \frac{\omega \tau}{2}$.

We can model a single lens system by having a translation of $M_{1}\left(t_{1}^{\prime}\right)$, where $t_{1}^{\prime}=t_{1}+\tau^{\prime} / 2$, followed by a thin lens of strength $\mathcal{C}$, followed by a translation of $M_{1}\left(t_{2}^{\prime}\right)$, where $t_{2}^{\prime}=t_{2}+\tau^{\prime} / 2$. The physical duration of the focusing is $T=t_{1}+t_{2}+\tau$, 
however the thin lens system has a total time $T^{\prime}=t_{1}+t_{2}+\tau^{\prime}=T-\tau+\tau^{\prime}$. For a single lens system, the condition $\mathcal{B}=0$ is met for the system $\mathcal{A B C D}$ matrix if we have:

$$
\mathcal{C} T^{\prime}=\frac{1}{\lambda(\lambda-1)}
$$

where

$$
\lambda=\frac{t_{1}^{\prime}}{T^{\prime}}
$$

yielding a magnification $(\lambda-1) / \lambda$. This formalism provides a useful way of designing a lens system and investigating its focusing properties. For a converging lens, equation (21) becomes:

$$
\omega T^{\prime} \sin \omega \tau=\frac{1}{\lambda(1-\lambda)}
$$

We consider an experimental situation where we fix the total time $T=212 \mathrm{~ms}$ and the maximum Amp-turns at $N I=10,000$ Amps. The geometry of the lens then fixes the maximum strength of the converging lens via $\omega_{r}{ }^{2}=\mu_{\mathrm{B}} B_{2} / m$. We can now solve to find an analytic result for $\lambda(\omega, \tau)$, which is illustrated in figure 4 for a single coil lens of radius $5 \mathrm{~cm}\left(\omega_{r}=69.6 \mathrm{rad} \mathrm{s}^{-1}\right.$ from equation (10) $)$. The $\lambda(\omega, \tau)$ parameter is maximised (and the magnification $(1-\lambda) / \lambda$ is minimised) when:

$$
1-\omega(T-\tau) \cot \omega \tau=0,
$$

which has the solution $\lambda=0.929$ at $\tau=23.7 \mathrm{~ms}$. This corresponds to a reduction in the atomic cloud size by a factor of -13.1 This is achieved when the pulse duration $\tau$ is from time $t=T-\tau$ to $t=T$, i.e. the lens pulse ends at the time of focus. Such focusing in three dimensions would increase the cloud density by more than 3 orders of magnitude. For a lens placed later in time, the magnetic pulse would not have finished at the predicted focal time $T$, resulting in an increase in cloud size at time $T$.

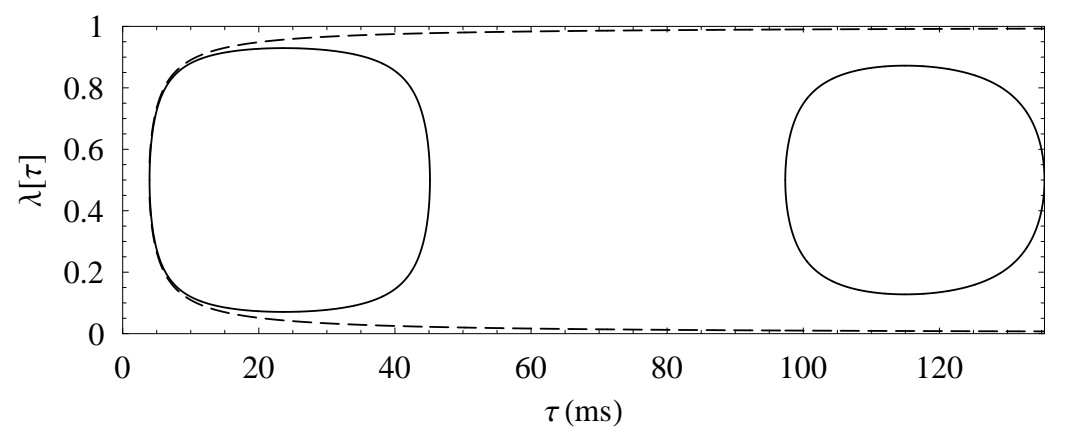

Figure 4. By fixing the strength of a $5 \mathrm{~cm}$ Strategy I radially converging lens, $\omega_{r}=69.6 \mathrm{rad} \mathrm{s}^{-1}$, and total experimental focusing time $T=212 \mathrm{~ms}$, we can vary the lens pulse width, $\tau$, and find the focusing parameter $\lambda(\tau)$ via equation (23). The impulse $\tau$ has a minimum of $3.9 \mathrm{~ms}$ at $\lambda=1 / 2$, and $\lambda$ is symmetric about this point. Also shown (dashed) is the result obtained if one makes the strong, short pulse approximation $\omega \tau \rightarrow 0$, leading to the simplification $\sin \omega \tau \approx \omega \tau$ in equation [23] - resulting unsurprisingly in a divergence for large pulse durations.

The above analysis would seem to suggest that the optimum strategy for achieving the smallest cloud size would be to construct a lens with a short, strong pulse $\omega \tau \rightarrow 0$, and use the latest possible pulse time $\lambda \rightarrow 1$. However, experimental constraints and lens aberrations alter the above conclusion. 


\section{Investigating various coil configurations for pulsed focusing}

\subsection{Methodology}

The $\mathcal{A B C D}$ matrix formalism outlined above is a useful starting point for studying pulsed magnetic focusing. However, this formalism ignores magnetic aberrations arising due to the departure of the real potential from an ideal parabolic spatial dependence (figure 3). For a non-parabolic potential the change in position and velocity which occur during lensing must be calculated numerically. Here, for the first time to our knowledge, we test the 'perfect' atomic lens approximations by performing numerical focusing simulations. The cloud and its motion are treated classically, and for the atomic densities encountered in the expanding cloud, the collision rate is negligible. The atoms travel on ballistic trajectories, except when a magnetic impulse is applied, in which case the full Stern-Gerlach force is included in the numerical integration. For the sake of definiteness we chose to investigate the focusing of ${ }^{85} \mathrm{Rb}$ atoms in an atomic fountain launched vertically through a height of $22 \mathrm{~cm}$ (which corresponds to a flight-time of $212 \mathrm{~ms}$ ), a height which is of interest experimentally. The atoms come to rest at the apex of their trajectories where they could be used for further experiments or loaded into a dipole trap. The effects of gravity were included, but these effects on the quality of focus were found to be negligible for the parameters used in these simulations.

The approach adopted was a numerical simulation, in which the trajectories of typically 500 atoms are followed. The initial velocity and position probability distributions are isotropically Gaussian for each Cartesian direction. The standard deviation of position was chosen to be a value typical of experiments at $0.4 \mathrm{~mm}$ [4], and the velocity distribution corresponding to a typical launch temperature obtained with $\mathrm{Rb}$ in moving molasses, namely $T=20 \mu \mathrm{K}$. These simulations facilitate the calculation of statistically relevant quantities, such as the standard deviation of the time-dependent size of the atomic cloud. In all the simulations the maximum current value in any coil was limited to $10,000 \mathrm{~A}$.

\subsection{Strategies I-III: axial/radial focusing}

To illustrate our methodology we discuss the focusing properties of a single, circular, current-carrying coil (Strategy I). Figure囵(a) shows the evolution of the $x$ component of a launched cloud of 500 atoms subject to a radially converging lens constructed from a single $5 \mathrm{~cm}$ radius coil. The impulse is applied half-way in time, and the length of the impulse is chosen to reverse the transverse velocity, as can be seen from the change in sign of the gradient after $t=106 \mathrm{~ms}$. For this case, the $\mathcal{A B C D}$ matrix predicts a radial focus with magnification -1 , which is in excellent agreement with the numerical simulation using a parabolic lens (using the strength of equation (10)). The vertical line at $t=212 \mathrm{~ms}$ corresponds to the imaging time. For real coils, it is seen that the focussed cloud image is significantly larger than the initial cloud. The aberration worsens as the coil radius decreases. Note that although focusing is seen in the radial direction, defocusing is seen in the axial direction due to the opposite sign of the magnetic field curvature.

Figure 5 (b) contains an analysis of the cloud in terms of shells of different radii measured from the centre of the coil; atoms further from the centre are not focussed as tightly, and also focus earlier in time. As the ratio between cloud extent and coil

radius decreases, the departure of the field from the parabolic approximation becomes 

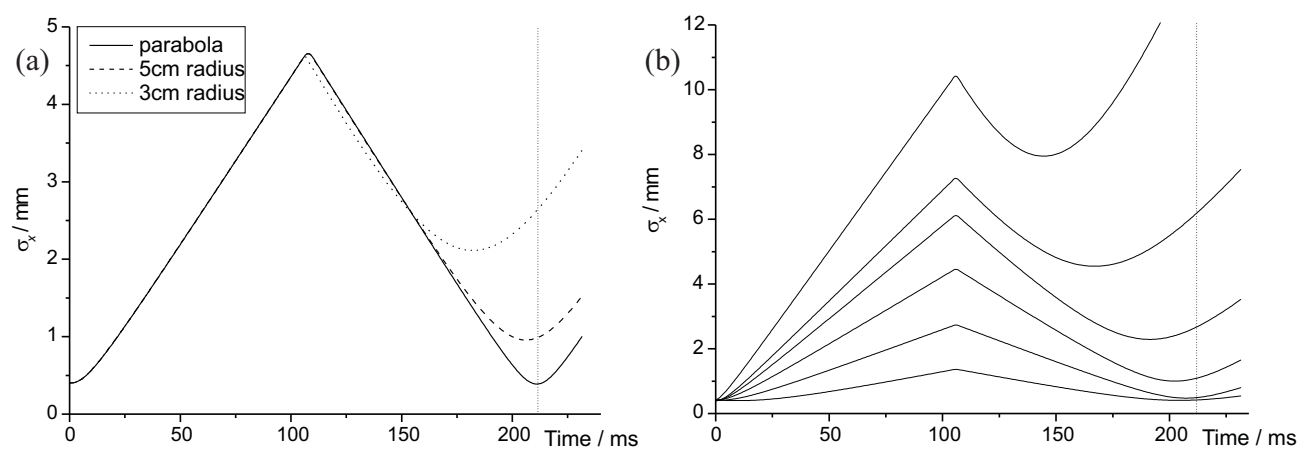

Figure 5. (a) Simulation of 500 atoms going through a 10,000 Amp-turn strategy I lens. The solid, dashed and dotted lines correspond to a parabolic approximation lens, a $5 \mathrm{~cm}$ radius lens, and a $3 \mathrm{~cm}$ radius lens respectively. The duration and timing of the impulse is chosen using the $\mathcal{A B C D}$ matrix formalism, bringing the atoms to a focus $22 \mathrm{~cm}$ above their launch height. (b) A shell plot of 1000 simulated atoms passing through a $3 \mathrm{~cm}$ radius strategy I lens. The distances from the coil centre are $0-10 \%$ of the coil radius, $10-20 \%$, through to $50-60 \%$. Atoms further from the centre are not focussed as well, and the focusing occurs at earlier times. Both of these factors degrade the image quality and size.

less significant. Therefore one method to reduce the aberrations experienced by the atoms is to increase the coil radius, or decrease the atomic cloud temperature.

We turn our attention to obtaining the minimum cloud size, equation (18), by investigating the effect of the $\lambda$ parameter. As discussed in section 3.1] a thin parabolic lens produces the smallest cloud size when $\lambda \rightarrow 1$, i.e. the pulse is applied as late in time as possible. This is a manifestation of Liouville's theorem - a more compact spatial extent can be generated at the expense of a larger velocity spread.

Figure 6 (a) shows simulations of radial focusing. The radial cloud expansion factor, $\sigma_{x} / \sigma_{x_{i}}$, is plotted as a function of the imaging parameter $\lambda$. It can be seen that the smallest cloud size for a parabolic lens occurs when one waits as long as possible before focusing, i.e. $\lambda$ is as close as possible to 1 (limited by the solution of equation (24)). This means that the magnetic impulse ends at the desired focusing time. For parabolic fits to the 3 and $5 \mathrm{~cm}$ radius single coils (Strategy I) the maximum values of $\lambda$ are 0.968 and 0.929 , corresponding to negative magnifications, $M=(1-\lambda) / \lambda$, of $1 / 30.1$ and $1 / 13.1$ respectively.

Along with the parabolic case, figure 6 (a) shows numerical simulations for 500 atoms passing through 3 and $5 \mathrm{~cm}$ radius coils, for both Strategies I (single coil) and II (two coils). As expected the $5 \mathrm{~cm}$ lens better approximates a parabola. Compared to the $\mathcal{A B C D}$ matrix result there is a marked difference in the behaviour of the minimum radially-focussed cloud-size for fields from real coils - the value of $\lambda$ at which the $\mathcal{A B C D}$ minimum is obtained is dominated by aberrations in the magnetic field. The $\mathcal{A B C D}$ matrix approach does not provide an adequate description of pulsed magnetic focusing when one considers the entire atomic cloud.

In figure 6 (b) the radial expansion factor of a $5 \mathrm{~cm}$ single coil (Strategy I) is plotted against time for values of $\lambda$ varying from 0.2 to 0.8 .

The easiest way to reduce aberrations appears to be the use of a very large coil radius. Unfortunately the curvature of the field decreases with the cube of the coil radius (equation (10), which necessitates longer pulse durations for larger radius 

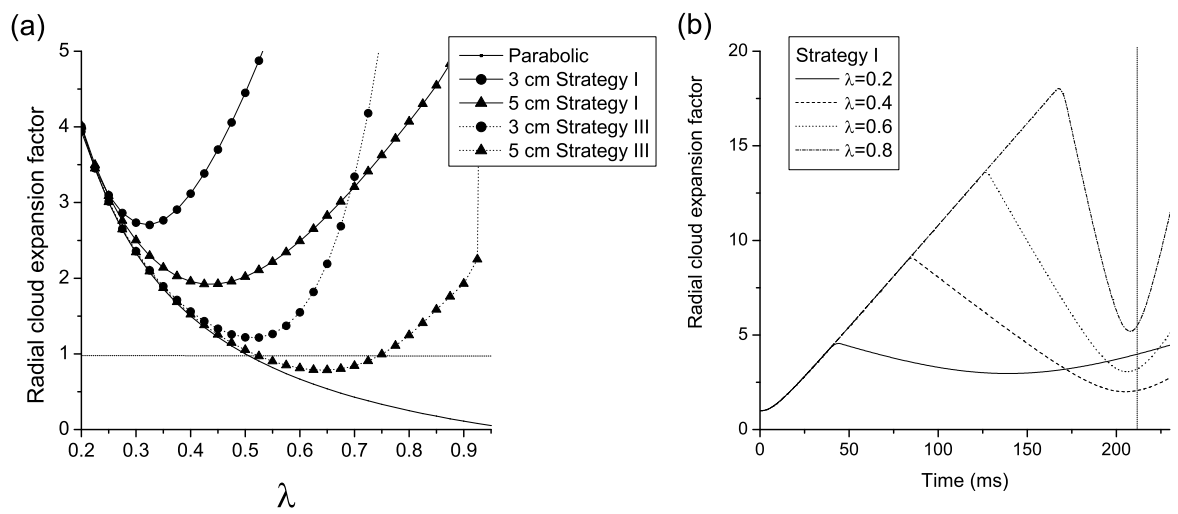

Figure 6. (a) The radial expansion factor, at $T=212 \mathrm{~ms}$, for radially converging lenses is plotted against $\lambda$. The solid line without symbols shows the parabolic lens solution, the solid (dotted) lines with symbols shows the result of atoms passing through Strategy I (II) lenses. Circular (square) symbols are used for the $3 \mathrm{~cm}(5 \mathrm{~cm})$ radius lenses. The Strategy II lens is more parabolic, allowing a smaller radial cloud size to be achieved: a cloud image 0.75 times the original size occurs for $\lambda=0.65$ using a $5 \mathrm{~cm}$ lens. (b) For a $5 \mathrm{~cm}$ Strategy I lens, the radial expansion factor is plotted against time for $\lambda$ values $0.2,0.4,0.6$ and 0.8 .

coils. This increase in pulse duration reduces the maximum value of $\lambda$ that can be used and therefore also limits the minimum cloud size. The aberrations can only be further reduced by increasing the current-turns, something which has experimental limitations.

The aberrations associated with a real coil dramatically affect the strategy for achieving a radially compact cloud. The impulse has to be applied significantly earlier than an $\mathcal{A B C D}$-matrix analysis would suggest. However, for experimentally-realistic parameters it is seen that it is possible to achieve a final radial cloud size that is smaller than the initial size. Strategy II (two coils of radius $a$ carrying the same current $I$, separated by $S=0.58$ ), clearly has a better performance than Strategy I (single coil), as seen in figure 6 (a). Most importantly, in the $5 \mathrm{~cm}$ case, the radial extent of the final cloud is 0.75 times the original radial extent of the cloud.

Strategy III produces an axially converging/radially diverging lens with a high level of harmonicity (figure 3) comparable to that of Strategy II. We omit our results for axial focusing in this paper, as it will be revisited in the context of alternategradient focusing in a future publication [19.

\subsection{Strategies $I V$-VI: isotropic $3 D$ focusing}

Isotropic 3D focusing can be achieved using two coils with differing currents (Strategy IV). For realistic experimental parameters, the numerical simulations showed that the aberrations in the lens smeared out any focusing. However, for unrealistically large lens radii and large currents (e.g. $15 \mathrm{~cm}$ and 200,000 Amp-turns) it is possible to achieve 3D focusing.

As discussed in section 2.3.3 a single coil can be made to have isotropic curvature 
(Strategy V). At $z= \pm \sqrt{2 / 7} a$, the axial and radial curvatures are equal, and the gradient of the field is non-zero. A numerical simulation was performed for a launched cloud, with an impulse applied when the cloud's centre of mass reached a distance $z=+\sqrt{2 / 7} a$ from the centre of a single coil. Due to the large departure from harmonicity for the experimentally realistic parameters we used, aberrations dominated and focusing was not observed.

The baseball lens (Strategy VI) yielded the best isotropic 3D lens. Figure [7 (a) shows the temporal evolution of the volume expansion factor, $\left(\sigma_{x} \sigma_{y} \sigma_{z} / \sigma_{x_{i}} \sigma_{y_{i}} \sigma_{z_{i}}\right)$, for a launched cloud subject to a focusing pulse from a baseball lens. Five different values of $\lambda$ are depicted, from 0.3 to 0.7 in steps of 0.1 . The bias coils have radii of $a=5 \mathrm{~cm}$, separation $S=15$, and current $N I=3082 \mathrm{~A}$; the baseball has sides of length $W=L=2$ and carries a $10,000 \mathrm{~A}$ current. The minimum cloud size is obtained when $\lambda=0.3$, and represents a 31.2 increase in cloud volume at the focal time, $T=212 \mathrm{~ms}$. This is to be contrasted with the 13,000 increase in cloud volume if no magnetic lens were used. It is interesting to note that for the parameters we have simulated, the results of a 'pure' Ioffe-Pritchard lens $(W=2, L \rightarrow \infty$ and $S=2)$ are almost identical to the baseball coil. Surprisingly the baseball lens performs better, and produces smaller cloud sizes at the focus.
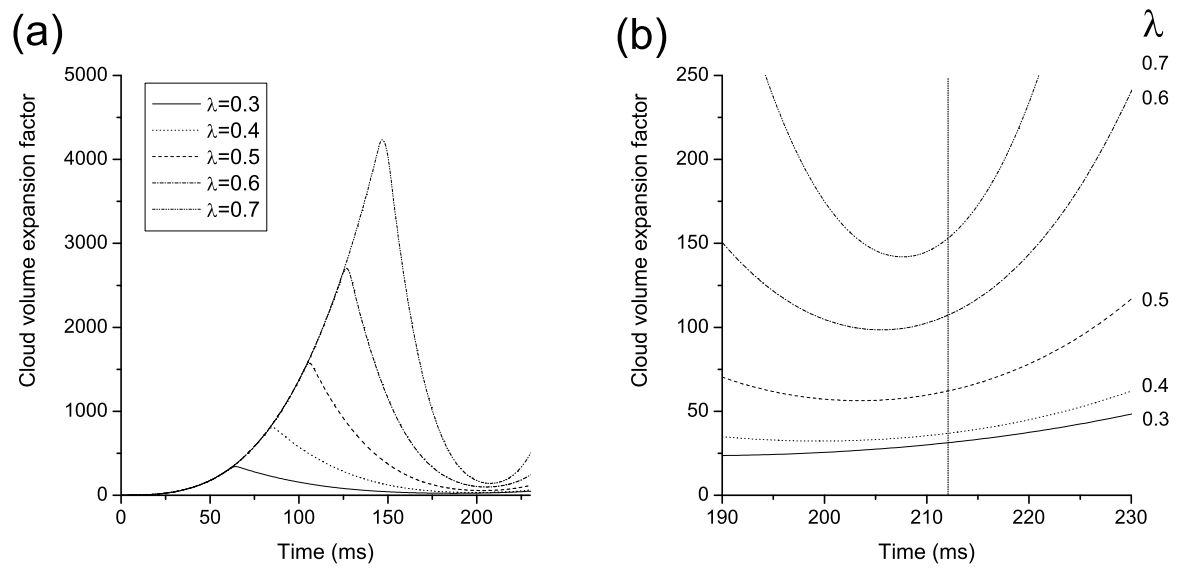

Figure 7. (a) A simulation of 500 atoms sent through an isotropic baseball coil lens (Strategy VI). The ratio of the cloud volume to the initial volume is plotted as a function of time for values of $\lambda$ ranging from 0.3 to 0.7. (b) A close-up of the simulations near the $t=T$ imaging time (vertical line).

\section{Discussion and Conclusion}

We have highlighted the limiting size of a focussed launched cold cloud of weakfield seeking atoms for various pulsed magnetic focusing techniques. The $\mathcal{A B C D}$ matrix formalism is convenient for giving an estimate as to the parameters needed for magnetic focusing, but does not contain the departure of the potential experienced by atoms from a perfect parabolic dependence for fields produced by real coils (and bars). In this work we have shown how important it is to consider these aberrations as they drastically alter the results. We have identified the origin of these aberrations, 
and described techniques for minimising them.

We have discussed single coil lenses (Strategy I), as well as five novel atomic lenses strategies (II-VI), and tested their aberrations both analytically and numerically. For all of the Strategies we found that our analytic results for aberrations (section 3) tied in well with the numerical simulations of section 4. It was demonstrated in Strategies II and III that a 'doublet' radially (axially) focusing lens formed from two coils with relative separation $S=0.58$ (2.63) provided much tighter focusing than the single-coil lens of Strategy I. Amongst the isotropic 3D lenses we found that the baseball lens (Strategy VI) was superior to the two coil lens of Strategy IV, which was in turn considerably better than the axially offset single coil lens of Strategy V. Of the singleimpulse lenses, the baseball lens offers the best possibilities for isotropically focusing a cloud of weak-field-seeking atoms in 3D. Experiments to test these predictions are underway in our laboratory.

It should be noted that in section 4 we have used the rms radius of a cloud of atoms to measure how tightly the entire atomic cloud is focused. By only considering a low-velocity fraction of the atomic distribution, even fractions as large as $50 \%$, it is possibly to reduce drastically the rms focal spot size, increasing the atomic density by orders of magnitude. We will address this complex issue in more detail in our future publication dealing with alternate-gradient focusing [19].

\section{Acknowledgments}

This work is supported by EPSRC, the UKCAN network and Durham University. We thank Charles Adams and Simon Cornish for fruitful discussions.

\section{References}

[1] Adams C S and Riis E 1997 Prog. Quant. Elec. 211

[2] Chu S 1998 Rev. Mod. Phys. 70 685; Cohen-Tannoudji C N 1998 Rev. Mod. Phys. 70 707; Phillips W D 1998 Rev. Mod. Phys. 70721

[3] Ramsey N F 1985 Molecular Beams 1st edition (Oxford, OUP)

[4] Hinds E A and Hughes I G 1999 J. Phys. D 32 R119

[5] Adams C S, Sigel M and Mlynek J 1994 Phys. Rep. 240143

[6] Roach T M, Abele H, Boshier M G, Grossman H L, Zetie K P and Hinds E A 1995 Phys. Rev. Lett. 75 629; Sidorov A I, McLean R J, Rowlands W J, Lau D C, Murphy J E, Walciewicz M, Opat G I and Hannaford P 1996 Quantum Semiclass. Opt. 8 713; Lau D C, Sidorov A I, Opat G I, McLean R J, Rowlands W J and Hannaford P 1999 Eur. Phys. J. D 5193

[7] Hughes I G et al. 1997 J. Phys. B: At. Mol. Opt. Phys. 30 647; ibid 30 2119; ibid 34 2869; Saba C V et al. 1999 Phys. Rev. Lett. 82468

[8] Kadio D, Houde O and Pruvost L 2001 Europhys. Lett. 54417

[9] Bloch I, Köhl M, Greiner M, Hänsch T W and Esslinger T 2001 Phys. Rev. Lett. 87030401 ; Arnold A S, MacCormick C and Boshier M G 2002 Phys. Rev. A 65 031601(R); Arnold A S, MacCormick C and Boshier M G 2004 J. Phys. B 37485

[10] Rosenbusch P et al. 2000 Phys. Rev. A 61 031404; Rosenbusch P et al. 2000 Appl. Phys. B 70 709.

[11] Meschede D and Metcalf H 2003 J. Phys. D 36 R17

[12] Szymaniec K, Davies H J and Adams C S 1999 Europhysics Letters 45450

[13] Müller D, Anderson D Z, Grow R J, Schwindt P D D and Cornell E A 1999 Phys. Rev. Lett. 83 5194; Dekker N H, Lee C S, Lorent V, Thywissen J H, Smith S P, Drndić M, Westervelt R M and Prentiss M 2000 Phys. Rev. Lett. 84 1124; Key M, Hughes I G, Rooijakkers W, Sauer B E, Hinds E A, Richardson D J and Kazansky P G 2000 Phys. Rev. Lett. 841371

[14] Folman R, Kruger P, Schmiedmayer J, Denschlag J and Henkel C 2002 Adv. At. Mol. Opt. Phys. 48263 
[15] Sauer J A, Barrett M D and Chapman M S 2001 Phys. Rev. Lett. 87 270401; Wu S, Rooijakkers W, Striehl P and Prentiss M 2004 Phys. Rev. A 70013409

[16] Cornell E A, Monroe C and Wieman C E 1991 Phys. Rev. Lett. 672439

[17] Maréchal E, Guibal S, Bossennec J L, Gorza M P, Barbé R, Keller J C and Gorceix O 1998 Eur. Phys. J. D 2 195; Maréchal E, Guibal S, Bossennec J L, Barbe R, Keller J C and Gorceix O 1999 Phys. Rev. A 594636

[18] Miossec T, Barbé R, Keller J-C and Gorceix O 2002 Opt. Commun. 209349

[19] Pritchard M J, Arnold A S, Smith D A and Hughes I G Pulsed magnetic focusing of launched cold atoms: II. Alternate gradient focusing in preparation

[20] Myrskog S H, Fox J K, Moon H S, Kim J B and Steinberg A M 2000 Phys. Rev. A 61053412

[21] Goldberg S, Strasser D, Heber O, Rappaport M L, Diner A and Zajfman D 2003 Phys. Rev. A 68043410

[22] Bethlem H B and Meijer G 2003 Int. Rev. Phys. Chem. 2273

[23] Dowling J P and Gea-Banacloche J 1996 Adv. At. Mol. Opt. Phys. 371

[24] Petrich W et al. 1995 Phys. Rev. Lett. 743352

[25] Earnshaw S 1842 Trans. Cambridge Philos. Soc. 797

[26] Smythe W R 1968 Static and Dynamic Electricity (New York, McGraw-Hill, 3rd edition) equations (5) and (6) p 271

[27] Bergeman T, Erez G and Metcalf H J 1987 Phys. Rev. A 351535

[28] Gott Y V, Ioffe M S and Tel'kovskii V G 1962 Nucl. Fusion Suppl. 3 1045; Pritchard D E 1983 Phys. Rev. Lett. 511336

[29] Ketterle W and Pritchard D E 1992 Phys. Rev. A 464051 